\title{
Management of Acute Vascular Injuries
}

\section{S. PONGPECH}

Section of Interventional Neuroradiology, Department of Radiology, Ramathibodi Hospital, Bangkok; Thailand

\section{Introduction}

Vascular injury of the head and neck region occurred not very frequent but can be potentially life treatening with very high morbidity and mortality, especially if prompt diagnosis and treatment decision are not accurately made on time. Mechanism of injury and pathophysilogy of the vascular wall damages of the patients were analysed including management of this group of patients.

\section{Material and Methods}

Patients with known traumatic vascular injury of the head and neck region in Ramathibodi hospital from 5/1995 to 5/2003 were analysed for duration from injuries, mechanism and sources of injury which could be both direct and indirect force, sources are from car accident, Bony fragments; bullet and iarthrogenic procedures.Pathophysiology changes are from dissection to false aneurysm and arterio-venous fistula. Methods of diagnosis and management decision and results were reviewed included some of the long term follow up.

\section{Results}

There are total 310 patients; $36(11.6 \%)$ of them had extracranial vascular injury; 261 $(84.2 \%)$ of them had base skull injury and 13 (4.2\%) of them had intracranial (intradural) injury. Within the extracranial group; there are
2 CCA, 20 ICA, 9 ECA, 5 VA). The base skull group are the major contribution and more than $90 \%$ of them are traumatic Carotid-cavernous fistula which all of them are from car accident. Multiple vascular injuries occurred in about $15 \%$ of all the patient. Pure intracranial vascular injury are rare.

Pathophysiolgy analysis showed majority of AVF followed by false aneurysm and dissection. Duration of injury were from 12 hours to 1.5 year (mostly within 3 months). Diagnosis and treatment decision were all done by senior interventional neuroradiologist; either by clinical diagnosis or with radiological investigation. Emergency treatment (within 6 hours after consultation) were performed in about $5 \%$ of the patient which all of them presented with either massive epistaxis or subarachnoid haemorrhage. Management decision ranged from close conservative follow up to mainly endovascular treatment and few cases of surgical management. There are $97 \%$ curative results (clinical and angiographical) of the management with $100 \%$ improvement of the clinical follow up (range from 3 months to 8 years)

\section{Conclusions}

Traumatic vascular lesions of the head and neck region; especially in acute phase (within 3 months) are the challenge conditions which could easily lead the patients to morbidity and mortality, but if prompt diagnosis and accurate 


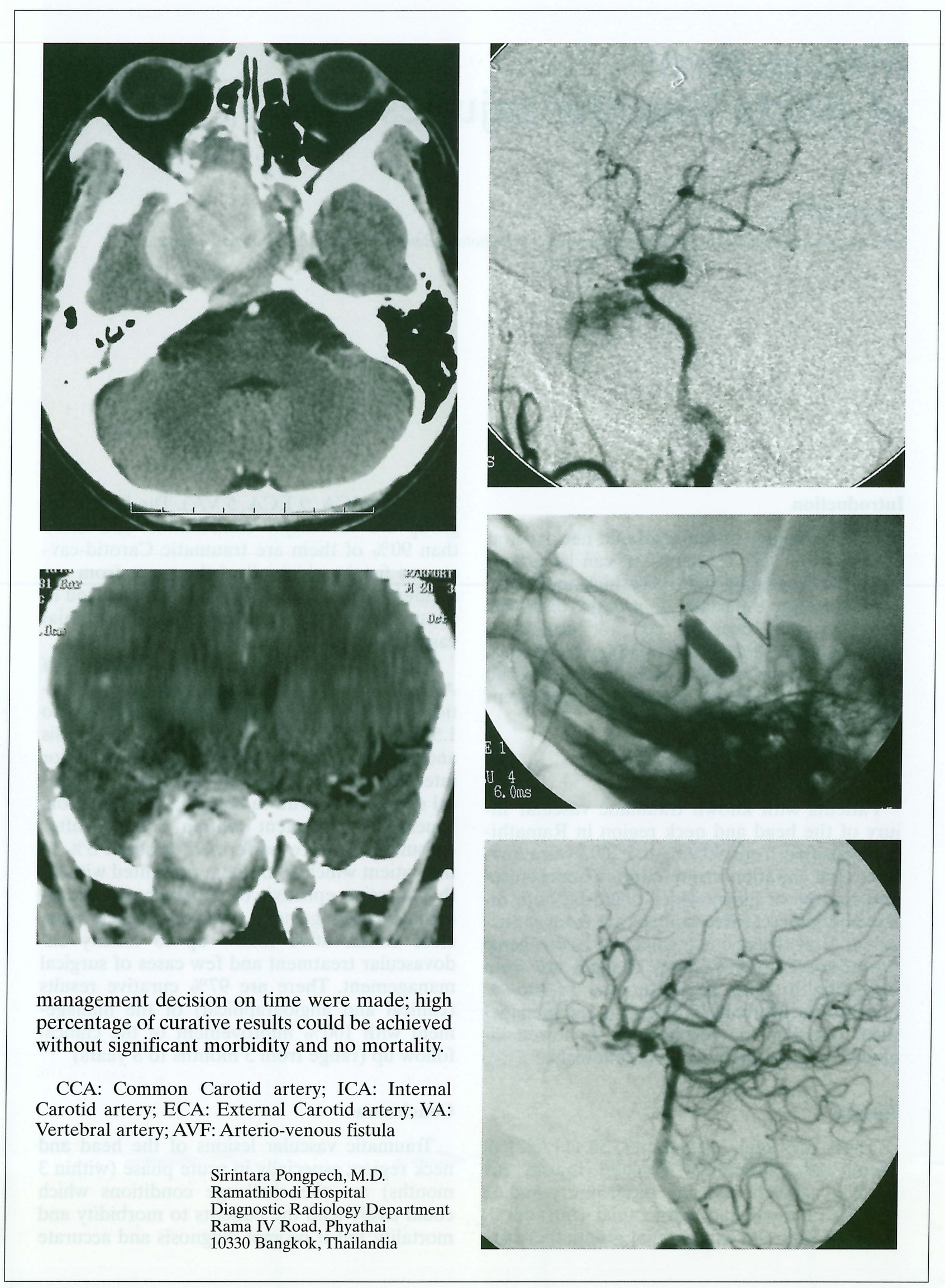

